\title{
Elements of Doping Engineering in Semiconductors
}

S.B. Zhang, S. Wei, and A. Zunger

National Renewable Energy Laboratory

Presented at the National Center for

Photovoltaics Program Review Meeting

Denver, Colorado

September 8-11, 1998

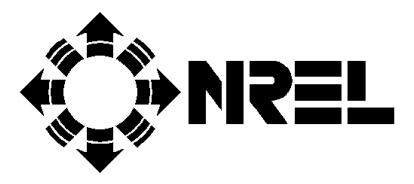

National Renewable Energy Laboratory 1617 Cole Boulevard

Golden, Colorado 80401-3393

A national laboratory of the U.S. Department of Energy Managed by Midwest Research Institute for the U.S. Department of Energy under contract No. DE-AC36-83CH10093

Work performed under task number PV902101

November 1998 


\begin{abstract}
NOTICE
This report was prepared as an account of work sponsored by an agency of the United States government. Neither the United States government nor any agency thereof, nor any of their employees, makes any warranty, express or implied, or assumes any legal liability or responsibility for the accuracy, completeness, or usefulness of any information, apparatus, product, or process disclosed, or represents that its use would not infringe privately owned rights. Reference herein to any specific commercial product, process, or service by trade name, trademark, manufacturer, or otherwise does not necessarily constitute or imply its endorsement, recommendation, or favoring by the United States government or any agency thereof. The views and opinions of authors expressed herein do not necessarily state or reflect those of the United States government or any agency thereof.
\end{abstract}

Available to DOE and DOE contractors from:

Office of Scientific and Technical Information (OSTI)

P.O. Box 62

Oak Ridge, TN 37831

Prices available by calling 423-576-8401

Available to the public from:

National Technical Information Service (NTIS)

U.S. Department of Commerce

5285 Port Royal Road

Springfield, VA 22161

703-605-6000 or 800-553-6847

or

DOE Information Bridge

http://www.doe.gov/bridge/home.html 


\title{
Elements of Doping Engineering in Semiconductors
}

\author{
S. B. Zhang, Su-Huai Wei, and Alex Zunger \\ National Renewable Energy Laboratory, Golden, Colorado 80401
}

\begin{abstract}
Using defect thermodynamics, we discuss physical factors that affect doping limits in semiconductors. The dependencies of the defect formation enthalpy on the atomic chemical potentials and on the electron Fermi energy are demonstrated. These dependencies, in particular on the Fermi energy, lead to spontaneous formation of charge-compensating defects that can limit doping. Experimental data compiled for III-V, II-VI, and I-III-VI 2 compounds support this view and further provide insight into the connections among different host materials. We argue that what matters is not the magnitude of the band gap that determines the dopability of a material, but rather, the relative position of the conduction-band minimum (in the case of n-doping) and the valence-band maximum (in the case of p-doping) with respect to vacuum.
\end{abstract}

\section{INTRODUCTION}

Semiconductors that cannot be doped are useless for most electronic and optoelectronic applications. Indeed, failure to dope a class of materials is often the single most important bottleneck for a semiconductor technology based on these materials. Overcoming this bottleneck can enable a whole new technology. Examples of past and present doping roadblocks include (i) p-type doping of wide-gap II-VI compounds for blue lasers, (ii) p-type doping of nitrides, (iii) the elusive ntype doping of diamond, (iv) p-type doping of (transparent conducting) oxides, and

(v) doping of alkali halides. Case (i) has been recently solved for ZnSe alone and case (ii) for GaN alone. All other cases remain unsolved.

There are three main modes of failure to dope:

(a) The desired impurity atom cannot be introduced into the host crystal because of limited solubility. Examples include large impurity atoms in small host crystals, or chemically inert impurities (Ba, rare gases) [1].

(b) The desired dopant is soluble in the host, but it produces a deep, rather than shallow level, so the impurity remains un-ionized at normal temperatures. Examples of such "localized centers" include $\underline{\mathrm{Cd} S}: \mathrm{Cu}$ or $\underline{\mathrm{ZnSe}} \mathrm{Cu}[2]$.

(c) The impurity atom is both soluble and ionizable, but as it produces free carriers, a spontaneously generated, oppositely charged native defect forms, which 
compensates and negates the effect of the intentional dopant. Examples include the DX center in Si-doped GaAlAs [3] and the AX center in N-doped ZnSe [4].

Categories (a) and (b) of "failure to dope" can sometimes be circumvented by changing the dopant. If $\mathrm{Ba}$ is insoluble in III-V compounds, one can attain $\mathrm{p}$ doping by using a smaller divalent cation such as $\mathrm{Zn}$. If $\mathrm{Cu}$ creates a deep level in ZnSe, one can use $\mathrm{N}$ that forms a shallower level [2]. However, category (c) of "failure to dope" is terminal, because it is not the chemical impurity that causes the failure to dope, but the free-carriers themselves. Thus, category (c) represents the true "doping limit" of a material. For example, no impurity or treatment has thus far resulted in any p-doping of main group oxides (e.g., $\mathrm{ZnO}, \mathrm{CaO}, \mathrm{MgO}$ ) or any n-doping of alkali halides or diamond.

In older literature, it was believed that such "doping limits" were caused by the very existence of a large band gap [5]. As evidence, it was customary to cite the fact that large-gap materials cannot be doped, e.g., GaN, diamond, and oxides. Today, it is clear that this is not the real story. We know that some large-gap materials can be doped, e.g., n-ZnO, n-CdS, and n-GaN. Surprisingly, however, doping can be strongly asymmetric with respect to holes and electrons, e.g., ZnO and $\mathrm{ZnS}$ can be doped only n-type, $\mathrm{CuInSe}_{2}$ can be doped both p- and n-type, $\mathrm{CuAlSe}_{2}$ cannot be doped, whereas $\mathrm{CuGaSe}_{2}$ and $\mathrm{CuInTe}_{2}$ can be doped only p-type. The existence of such a pronounced asymmetry cannot be explained simply by the existence of a large gap. We need another explanation.

The rich literature on doping of semiconductors and insulators shows, surprisingly, that each case of "failure to dope" was treated in the literature as an isolated issue. Thus, the literature on the failure to p-dope $\mathrm{ZnSe}$ was divorced from the literature on the failure to n-dope diamond or from the literature on doping difficulties in nitrides and carbides. It appears that there is a need to study the "science of failure-to-dope" as a generic discipline. Our preliminary studies, described below, show that indeed there are common and surprisingly simple principles that cut across failure to dope in different material classes. We find that failure to dope is not related to the size of the band gap per-se, but rather, to the position of the valence-band maximum $(\mathrm{VBM})$ with respect to the p-like pinning energy $E_{p i n}^{(p)}$, and the position of the conduction-band minimum (CBM) with respect to the n-like

pinning energy $E_{p i n}^{(n)}$. This opens a new strategy for doping. Here, we discuss some of the elements of such a science.

\section{ELEMENTS OF THERMODYNAMICS OF DOPING}

A key realization regarding doping is that the formation enthalpy of an impurity A in a solid depends on the chemical potentials, $\mu_{\alpha}$, of atom $\alpha$ and on the electron Fermi level, $E_{F}$. In the next two subsections, we describe these dependencies. 


\section{Dependence of Formation Energies on Chemical Potentials}

The energy of introducing a charge-neutral impurity $A^{0}$ into a crystal depends on the atomic chemical potential of the impurity A. This is because the impurity is taken from a given "reservoir" having a finite energy, $\mu_{A}$. The formation enthalpy of the impurity is thus

$$
E\left(A^{0}\right)=E_{t o t}\left(A^{0}\right)-E_{t o t}(0)-\mu_{A}+\mu_{\text {host }},
$$

where $E_{\text {tot }}\left(A^{0}\right)$ is the total energy of the host crystal having one impurity; $E_{\text {tot }}(0)$ is the total energy of the host without any impurity; $\mu_{A}$ is the energy of the impurity in the reservoir from which it is taken, e.g., atomic or molecular gas of the impurity atoms; and, in the case of a substitutional impurity, $\mu_{\text {host }}$ is the energy of the host atom in its respective reservoir. Consider a Ga impurity substituting for a Si atom in an Si host crystal. One has $\mathrm{A}=\mathrm{Ga}$ and host $=\mathrm{Si}$. In thermal equilibrium, the reservoir energy for impurity $G a_{S i}$ cannot be higher than the energy of pure solid $\mathrm{Ga}$ (otherwise, Ga will leave the Si host and precipitate as a solid). Furthermore, thermal equilibrium requires that $\mu_{\text {host }}$ equals the energy of the solid Si (otherwise, the Si host will either grow or shrink, contradicting the equilibrium assumption). For convenience, we will set the energies of all elemental solids (or molecules, in the case of nitrogen or oxygen) to zero; so, in addition to Eq. (1), we have the following restrictions on the atomic chemical potentials

$$
\begin{gathered}
\mu_{A} \leq 0 \\
\mu_{\text {host }}=\Delta H_{\text {host }},
\end{gathered}
$$

where $\Delta H_{\text {host }}$ is the formation enthalpy of the host. For binary crystals such as GaAs or ZnSe, the host is made of cation $(\mathrm{C})+$ anion $(\mathrm{X})$, and the chemical potentials $\mu_{C}$ and $\mu_{X}$ must satisfy

$$
\mu_{C}+\mu_{X}=\Delta H_{\text {host }},
$$

where $\Delta H_{\text {host }}$ is the formation enthalpy of the binary CX. In analogy with the inequality in Eq. (2), we have for binaries

$$
\mu_{A} \leq 0 ; \mu_{C} \leq 0 ; \mu_{X} \leq 0
$$

otherwise, precipitates of the elemental solids will form. In the discussion of binary compounds, it is a common practice to consider only $\mu_{C}$ from which $\mu_{X}$ is determined by Eq. (3). Using Eqs. (3) and (4), one then finds that $\mu_{C}$ is bounded by

$$
\Delta H_{\text {host }} \leq \mu_{C} \leq 0
$$

Native defects are a special case, where the defect formation enthalpy $E\left(D^{0}\right)$ is only a function of $\mu_{C}$. 
We conclude with Fig. 1, which shows [6] an example of calculated formation enthalpies of a few native defects in p-type GaAs. We see that the Ga-on-As antisite and the As vacancy are easy to form in Ga-rich conditions, whereas in Asrich conditions, the As-on-Ga antisite and the Ga vacancy are easy to form instead. For a detailed description and discussion of the chemical potential dependence of the defect formation enthalpies in ternaries such as $\mathrm{CuInSe}_{2}$, we refer the readers to Ref. [7].

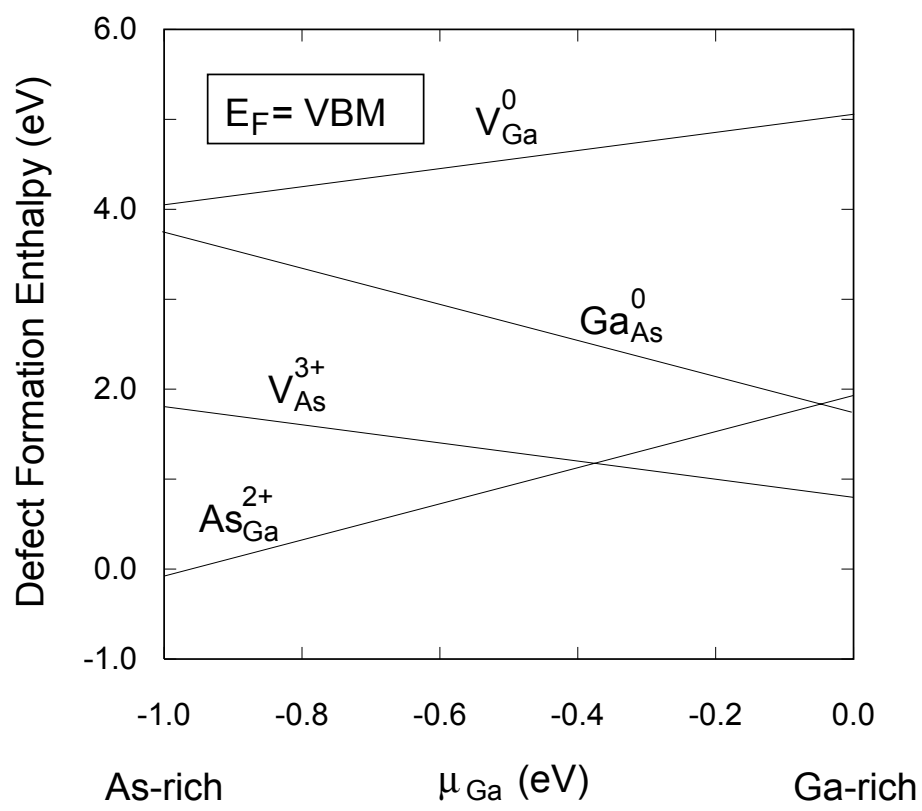

FIGURE 1. Calculated point-defect formation enthalpy in GaAs as a function of $\mu_{G a}$.

\section{Dependence of Defect Formation Enthalpy on the Fermi Level}

The energy of introducing a neutral impurity $A^{0}$ into a crystal does not depend on $E_{F}$. However, the energy of introducing a positively charged impurity $A^{+}$equals the energy of introducing a neutral impurity $A^{0}$, minus the energy $\mathrm{E}(0 /+)$ needed to ionize $A^{0}$ to form $A^{+}$, plus the energy of the ionized electron. Because this electron resides in the Fermi reservoir, its energy is $E_{F}$ :

$$
E\left(A^{+}\right)=E\left(A^{0}\right)-E(0 /+)+E_{F} .
$$

For a double donor, we will have $+2 E_{F}$. Thus, as Fig. 2 shows, the energy needed to form $A^{+}$increases as the Fermi energy increases. So, donors (that produce electrons in the reaction $A^{0} \rightarrow A^{+}+e$ ) are more difficult to form in electron-rich (n-type) materials. Similarly, for acceptors, the formation enthalpy decreases as $E_{F}$ increases: 


$$
E\left(A^{-}\right)=E\left(A^{0}\right)+E(-/ 0)-E_{F} .
$$

So, acceptors (that produce holes in the reaction $A^{-}+h \rightarrow A^{0}$ ) are more difficult to form in hole-rich (p-type) materials.

These simple considerations show the following:

(a) If we dope a material intentionally n-type via some donor impurity, as $E_{F}$ moves up in the gap, the formation enthalpy of native acceptors $E\left(A^{-}\right)$decreases. At some point, the formation enthalpy is so low that such native acceptors (e.g., cation vacancy or DX centers) could form spontaneously, thus negating the effect of the intentionally introduced donors.

(b) If we dope a material intentionally p-type via some acceptor impurity, as $E_{F}$ moves down in the gap, the formation enthalpy of native donors $E\left(A^{+}\right)$decreases. At some point, the formation enthalpy is so low that such native donors (e.g., the AX center) could form spontaneously, thus negating the effect of the intentionally introduced acceptors.

Thus, the position of the Fermi level decides which native defect will form spontaneously, and thus, halt doping.

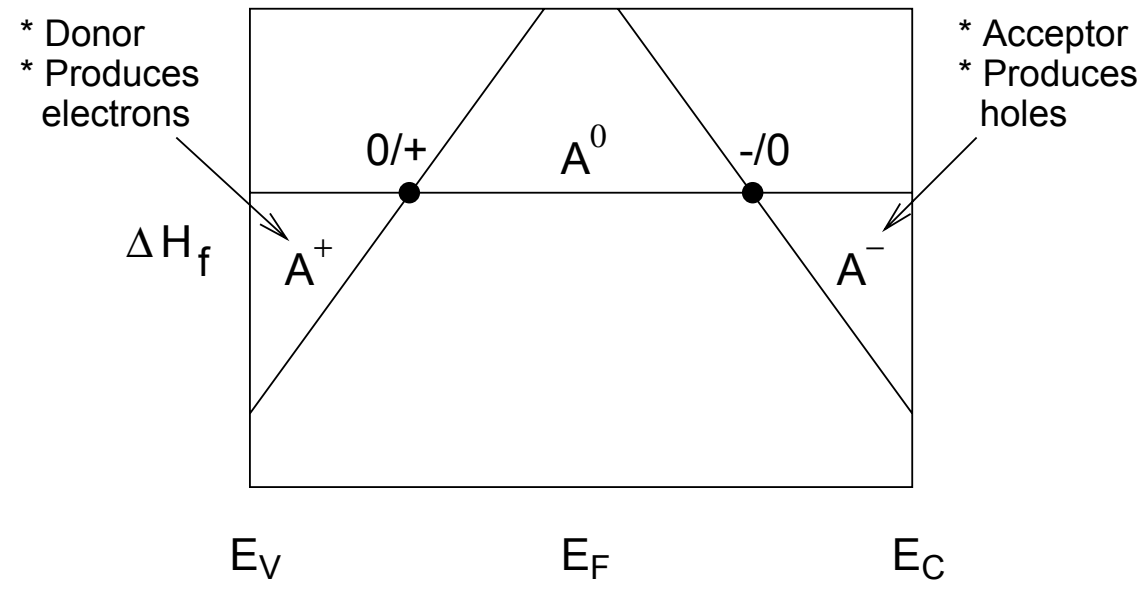

FIGURE 2. Schematic change of the defect formation enthalpies as a function of $E_{F}$.

\section{Determination of the Fermi Energy at Which Doping Stops}

We can now calculate empirically the value of $E_{F}$ at which doping stops, hoping that these values of $E_{F}$ will reveal some interesting regularities and physics. Doping stops when there are enough spontaneously generated defects to compensate the intentional dopants. The net concentration $N^{(n / p)}\left(T, E_{F}\right)$ of free carriers (electrons or holes) in a semiconductor is determined, in the single, parabolic band approximation, by the position of the Fermi energy via the Fermi-Dirac integral,

$$
N^{(\mathrm{n} / \mathrm{p})}\left[T, E_{F}^{(n / p)}\right]=\frac{1}{2 \pi^{2}}\left[2 m^{*,(\mathrm{n} / \mathrm{p})}\right]^{3 / 2} \int_{0}^{\infty} \frac{E^{1 / 2} d E}{\exp \left[\beta\left(E-E_{F}^{(n / p)}\right)\right]+1},
$$


where $\beta=1 / k T$ is the temperature factor, and $\mathrm{m}^{*}$ is the appropriate effective mass. If we know the measured maximum electron or hole concentration, $N_{\max }^{(n / p)}$, we may obtain an estimate of the values of the upper and lower bounds for $E_{F}$, i.e., $E_{\text {pin }}^{(n)}$ and $E_{\text {pin }}^{(p)}$, simply by inverting Eq. (8).

We plotted the calculated values of $E_{\text {pin }}^{(n)}$ and $E_{\text {pin }}^{(p)}$ with the VBM set at the same energy for all III- $V$ compounds. Although the data for $E_{p i n}^{(p)}$ are scattered within a relatively small range of $0.5 \mathrm{eV}$, the data for $E_{p i n}^{(n)}$ are scattered over a wide range of $1.2 \mathrm{eV}$, showing no emerging trends.

A similar problem of absence of the apparent trends in energy levels existed in another field, namely, that of transition-metal impurities in semiconductors [8]. It was known for a long time that if one refers the gap level of a given impurity (e.g., $\mathrm{Co}$ ) in different hosts (e.g., ZnS, ZnSe, CdSe) to the valence-band maximum of the hosts, no clear trend emerges. However, Caldas, Fazzio and Zunger [8] showed that if one refers the impurity levels to the vacuum level, the states of a given impurity in different host all line up.

Thus, following Walukiewicz [9], we will do the same for our calculated p-type and n-type Fermi energy pinning levels. However, now we will use modern, calculated values of unstrained band offsets [10] that are believed to have correct chemical trends. This is shown in Fig. 3 for III-V compounds. Except for p-type GaSb, both $E_{\text {pin }}^{(p)}$ and $E_{p i n}^{(n)}$ are within approximately a half eV range. Results for II-VI and I-III- $\mathrm{VI}_{2}$ ternary compounds are shown in Fig. 4. Now we can see clear chemical trends, which are reviewed next.

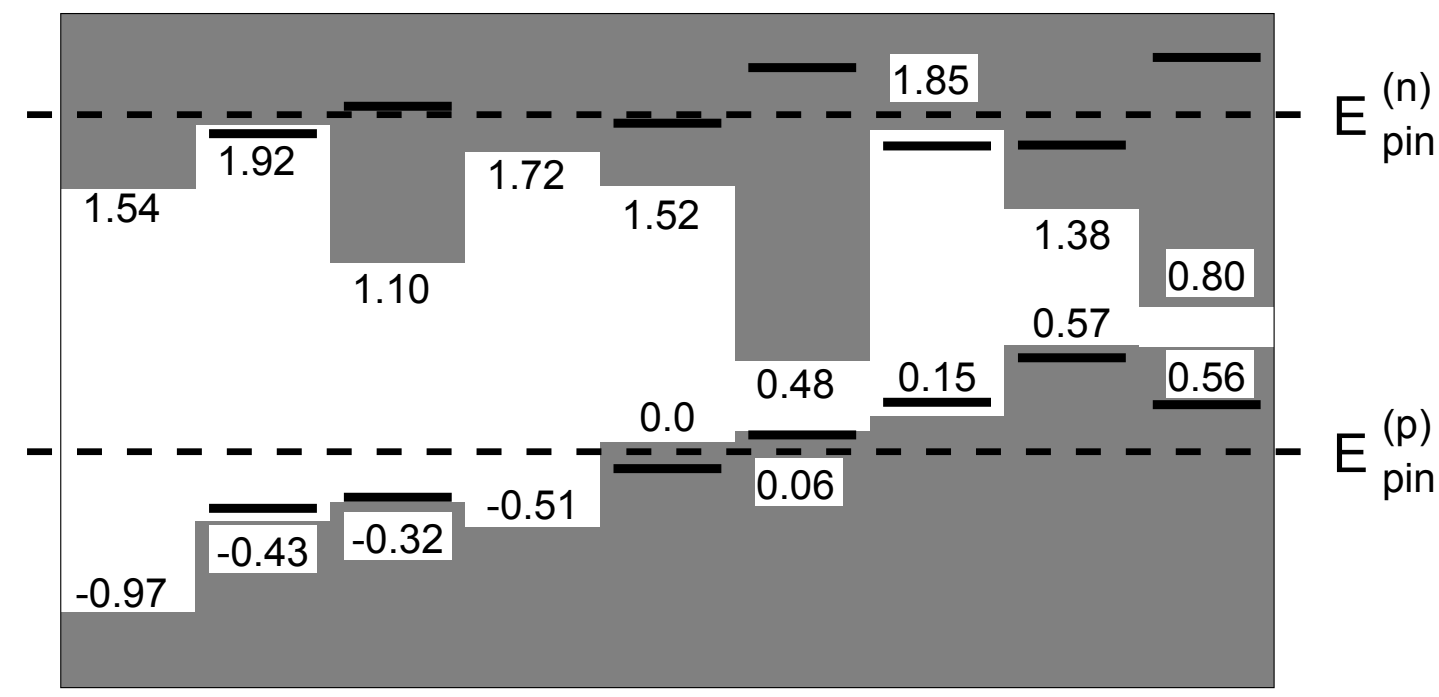

\section{AIP GaP InP AIAs GaAs InAs AISb GaSb InSb}

FIGURE 3. Calculated pinning levels for III-V compounds. Band line-up includes the calculated valence band offsets. Position of the $E_{\text {pin }}$ 's are indicated by the dash lines. 


\section{The Emerging Phenomenology of Doping Limits}

We see from Figs. 3 and 4 that there are two empirical pinning energy levels, $E_{p i n}^{(n)}$ and $E_{p i n}^{(p)}$, common to III-V compounds, and separately to all the II-VI compounds and separately to the I-III- $\mathrm{VI}_{2}$ compounds, that determine when doping stops. We can formulate the "doping limit rules":

(a) If $E_{C B M}>>E_{p i n}^{(n)}$, the material cannot be doped n-type;

(b) if $E_{V B M}<<E_{p i n}^{(p)}$, the material cannot be doped p-type.

What this implies is the following:

(i) Materials that can be doped n-type must have a low enough CBM, i.e., a large electron affinity, $\chi$. Good n-type materials are InP, InAs, InSb, ZnO, CdS, CdSe, and CdTe. However, n-type doping is not possible in $\mathrm{CuGaSe}_{2}, \mathrm{CuAlSe}_{2}$, and $\mathrm{CuInTe}_{2}$.

(ii) Materials that can be doped p-type must have a high enough VBM, i.e., a small workfunction, $\Phi$. Good p-type materials are $\mathrm{InSb}, \mathrm{CuInTe}_{2}$, and $\mathrm{ZnTe}$. It is not possible to dope $\mathrm{ZnO}$ and $\mathrm{ZnS}$ p-type.

(iii) To make a p-type transparent conducting oxide, one needs to push up the

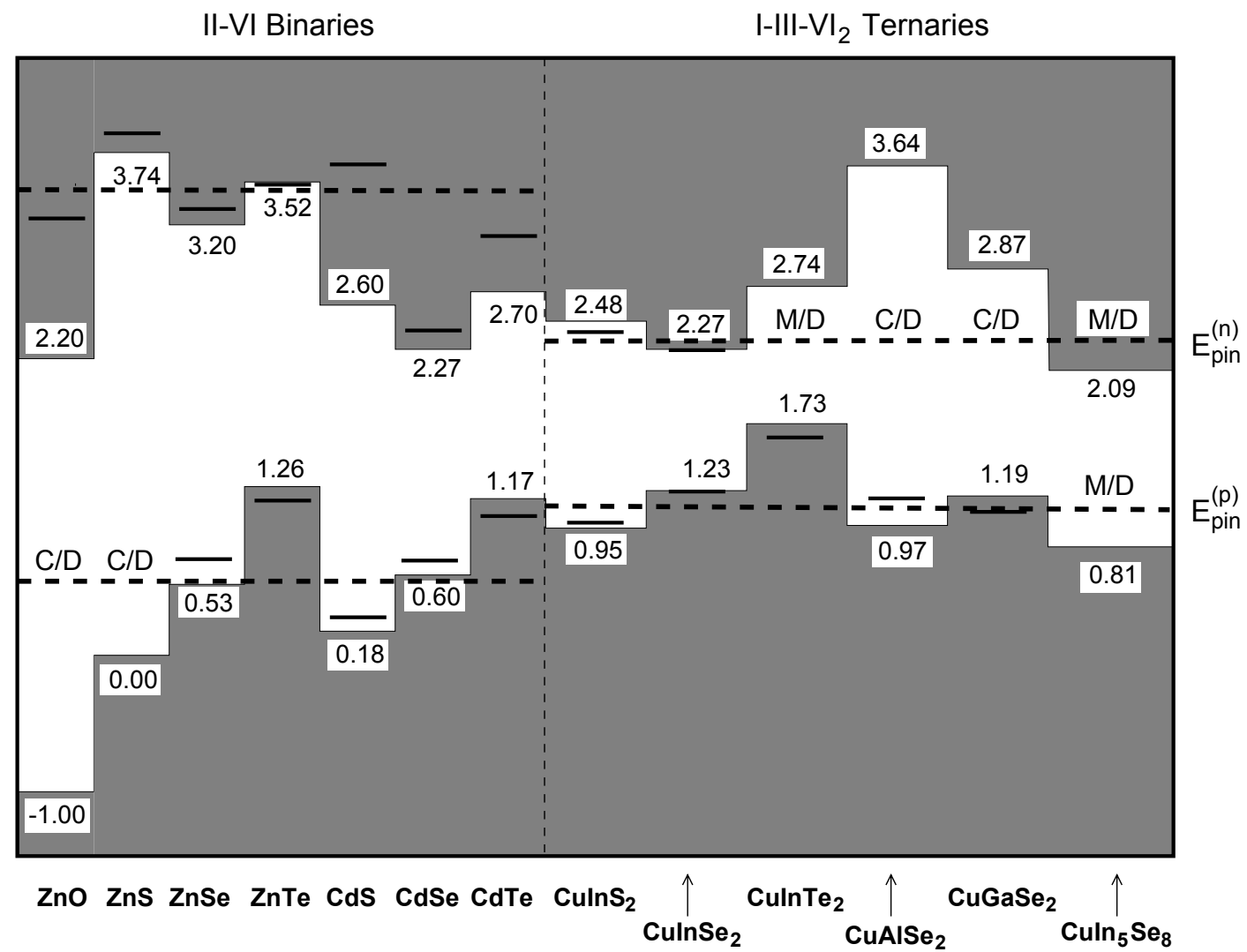

FIGURE 4. Calculated pinning levels for II-VI and I-III-VI ${ }_{2}$ compounds. C/D denotes "consistent with data" whereas M/D denotes "missing data". 
VBM. For example, one might replace oxygen by sulphur or substitute Zn by active d-electron cations such as a transition metal. The repulsion between anion $\mathrm{p}$ and transition metal $d$ near the Brillouin zone center will shift the anion-p VBM to higher energy, which will reduce $\Phi$ and enable p-doping.

\section{SUMMARY}

Based on basic thermodynamics, we discussed the key elements that lead to spontaneous formation of charge-compensating defects that limit doping. The dependencies of the defect formation enthalpy on the atomic chemical potentials and on the electron Fermi energy are emphasized. It is the dependence of the defect formation enthalpy on the Fermi energy that eventually leads to spontaneous formation of charge-compensating defects and the pinning of the Fermi energy. The two pinning levels are calculated using compiled experimental data for III-V, II-VI, and I-III-VI $\mathrm{V}_{2}$ compounds. This suggests that the intrinsic defects leading to doping limitations have some universal feature, at least within a well-defined group of solids (e.g., III-V compounds). We find that it is not the magnitude of the band gap that determines the dopability of a material, but rather, it is the positions of the CBM (in n-type) and the VBM (in p-type) relative to vacuum. The atomic origin of the doping limitations can be resolved in the future by first-principles calculations and experiments. This analysis points to new strategies for designing ways to overcome doping limits.

\section{ACKNOWLEDGEMENT}

This work was supported by the U. S. Department of Energy, DOE-EE under Contract No. DE-AC36-83CH10093.

\section{REFERENCES}

1. V. Singh and A. Zunger, Phys. Rev. B25, 907 (1982).

2. A. Zunger, in "Solid State Physics" edt. H. Ehrenreich and D. Turnbull (Academic Press, New York), 39, 275 (1986).

3. D. J. Chadi and K. J. Chang, Phys. Rev. Lett. 61, 873 (1988).

4. C. H. Park and D. J. Chadi, Phys. Rev. Lett. 75, 1134 (1995).

5. J. Van Vechten, in Handbook of Semiconductors edt. T. S. Moss (North Holland, Amsterdam, 1980), 3, p. 3.

6. S. B. Zhang and J. E. Northrup, Phys. Rev. Lett. 67, 2339 (1991).

7. S. B. Zhang, S.-H. Wei, A. Zunger, and H. Katayama-Yoshida, Phys. Rev. B57, 9642 (1998).

8. M. Caldas, A. Fazzio, and A. Zunger, Appl. Phys. Lett. 45, 671 (1984).

9. W. Walukiewicz, J. Vac. Sci. Technol. B5, 1062 (1987).

10. S.-H. Wei and A. Zunger, Appl. Phys. Lett. 72, 2011 (1998). 